\title{
Validation of a Novel Method for Cardiac Output Estimation by CT Coronary Angiography
}

\author{
Hetal H. Mehta ${ }^{1}$, Brian G. Choi ${ }^{1}$, Reza Sanai ${ }^{1}$, Raman S. Dusaj ${ }^{1}$, Amr Mohsen ${ }^{2}$ \\ Chunlei Liang ${ }^{2}$, Jannet F. Lewis ${ }^{1}$, Robert K. Zeman ${ }^{3}$ \\ ${ }^{1}$ Department of Medicine, The George Washington University, Washington, DC, USA \\ ${ }^{2}$ Department of Mechanical and Aerospace Engineering, The George Washington University, Washington, DC, USA \\ ${ }^{3}$ Department of Radiology, The George Washington University, Washington, DC, USA \\ Email: bchoi@gwu.edu
}

Received July 7, 2012; revised August 19, 2012; accepted September 11, 2012

\begin{abstract}
Background: Cardiac output can be estimated during retrospectively gated CT coronary angiography by anatomically determining left ventricular volumes; prospective triggering to minimize radiation precludes this methodology. We propose an alternative method for cardiac output estimation based on preclinical models suggesting that cardiac output may be inversely related to contrast washout from the aortic root during timing bolus scanning, as measured by peak aortic root contrast attenuation. Methods: 34 patients had CT coronary angiography timing bolus performed with $20 \mathrm{ml}$ iodixanol at $5.5 \mathrm{ml} / \mathrm{s}$ followed by $20 \mathrm{ml}$ normal saline at $5.5 \mathrm{ml} / \mathrm{s}$ through an 18-Ga antecubital catheter. Peak aortic root contrast attenuation was correlated to cardiac output calculated by echocardiography using heart rate stroke volume from biplane Simpson's method. Results: Mean age was $58 \pm 13$ years; body surface area, $2.0 \pm 0.5 \mathrm{~m}^{2} .53 \%$ were women. Stroke volume, cardiac output and cardiac index were $67 \pm 19 \mathrm{ml}, 4.5 \pm 1.6 \mathrm{~L} / \mathrm{min}$, and $2.2 \pm 0.7 \mathrm{~L} / \mathrm{min} / \mathrm{m}^{2}$, respectively. Peak aortic root contrast attenuation was $207 \pm 46 \mathrm{HU}$ and correlated to cardiac output and cardiac index with $r=-0.64, p<0.0001$ and $r=-0.55, p<0.001$, respectively. Regression analysis estimates cardiac output $=-0.02$ peak aortic root contrast attenuation +9.1 . Conclusion: This novel method for cardiac output estimation by CTCA appears feasible. The CT physiologic parameters using the timing test-bolus data moderately correlated with echocardiographic assessment of cardiac output. The calculation of cardiac output adds important hemodynamic data to anatomic information provided by CTCA, and further development of this method may preserve assessment of left ventricular performance in prospective triggering.
\end{abstract}

Keywords: Cardiac Output; Coronary CT Angiography; Hemodynamics; Prospective Triggering; Validation

\section{Introduction}

The assessment of cardiac output (CO) is an integral component of cardiovascular evaluation. The gold standard is invasive right-heart catheterization, but non-invasive imaging modalities include echocardiography, cardiac magnetic resonance imaging, and cardiac computed tomography (CT) [1,2]. CT coronary angiography (CTCA) uses electrocardiogram-gated image acquisition after contrast administration when a predetermined level of contrast opacification is reached to obtain high-resolution, three-dimensional depictions of both the cardiac structures and the coronary vessels [3]. Depending on the scanner, two acceptable methods exist: automatic bolus tracking or the timing bolus method for determining the contrast transit time. The entire CT data set, synchronized to the electrocardiogram (EKG), is acquired using either one of two different modes: retro spective gatingor prospective triggering. Retrospective EKG gating mode is performed with helical acquisition (patient/table is continuously advanced during the gantry rotation) with the $\mathrm{X}$-ray tube turned on throughout the entire cardiac cycle. Data from the most motion-free phase (diastasis usually occurs in mid-diastole) are then reconstructed for analysis. By contrast, prospective EKG triggering involves axial acquisition (table intermittently advances, usually with each heart beat) with the X-ray tube on during diastasis only [3].

To determine CO by CT, retrospective EKG gating is most commonly used for anatomic assessment of the left ventricular volumes. Recent emphasis on radiation minimization [4], however, has resulted in more frequent use of prospective triggering. Anatomic methods for calculation of CO cannot be applied to prospectively triggered images, since these images are obtained only during diastole. Therefore, it would be desirable to retain easily 
the ability to assess hemodynamic information from prospectively triggered CT coronary angiography (CTCA) in addition to evaluating the coronary arteries.

Cardiac output by invasive catheterization can be determined by the thermodilution method [5], in which the temperature change resulting from the injection of cold saline at a proximal catheter port as measured from its distal end is assessed. Such methodology has been replicated determining a contrast-based time-enhancement curve by gamma variate to exclude recirculation and cardiac output determination by the Stewart-Hamilton equation [6-8], but a simpler calculation that does not require dedicated software tools would be advantageous for adoption. Analogous to this method, we hypothesized that CO may be inversely to peak aortic root contrast attenuation (PARCA) as faster contrast washout from the aortic root during timing bolus scanning may occur with higher output, as suggested by preclinical study [9]. To determine the feasibility and validate this hypothesis, PARCA and other physiologic parameters derived form the timing test-bolus data were correlated with echocardiographic assessment of CO.

\section{Methods}

\subsection{Study Cohort}

We conducted a retrospective analysis of patients who underwent both echocardiography and CTCA within the same year at a tertiary academic medical center between January 2007 and May 2010.

Subjects included in the study had no documented change in clinical status between echocardiography and CT $(n=34)$. Three CT studies with poor acquisition of images (e.g., breathing during timing bolus acquisition) were excluded from the study. If multiple echocardio grams were present for the same patient, the echocardiogram closest in temporal proximity to the CT was chosen. Charts were reviewed for demographic information, including age, gender, height and weight, and the CTCA indication. The institutional review board at the center approved the study, and the need for written informed consent was waived.

\subsection{CTCA Protocol}

Patients underwent CTCA performed with 64-row multidetector CT (GE Lightspeed VCT, GE Healthcare; Waukesha, Wisconsin) using a timing test-bolus method to optimize image acquisition $(120 \mathrm{kV}, 100 \mathrm{~mA}, 5 \mathrm{~mm}$ slice thickness). All patients were in sinus rhythm and treated with metoprolol tartrate (American Regent Laboratories; Shirley, New York) to achieve a resting heart rate less than 65 beats per minute. Timing bolus was performed with $20 \mathrm{ml}$ iodixanol (Visipaque, GE Healthcare; Cork, Ireland) at $5.5 \mathrm{ml} / \mathrm{s}$ followed by $20 \mathrm{ml}$ normal saline (Braun; Irvine, California) at $5.5 \mathrm{ml} / \mathrm{s}$ through an 18-Ga antecubital catheter. The timing test-bolus images were performed using a low-milliamperage-second technique, beginning at 8 seconds and concluding at 34 seconds after the initiation of contrast injection, with images obtained every 2 seconds at the level of the aortic root during end-inspiratory breath hold. A representative series of images is shown in Figure 1.

Subsequent diagnostic scanning was performed applying the timing bolus results. Prospectively-triggered CCTA ( $0.625 \mathrm{~mm}$ collimation) was performed using a tube voltage of $120 \mathrm{kVp}$ and a weight-based determination of $\mathrm{mA}$. For the purposes of this study only the timing test-bolus data was evaluated, and correlated with echocardiographic data.
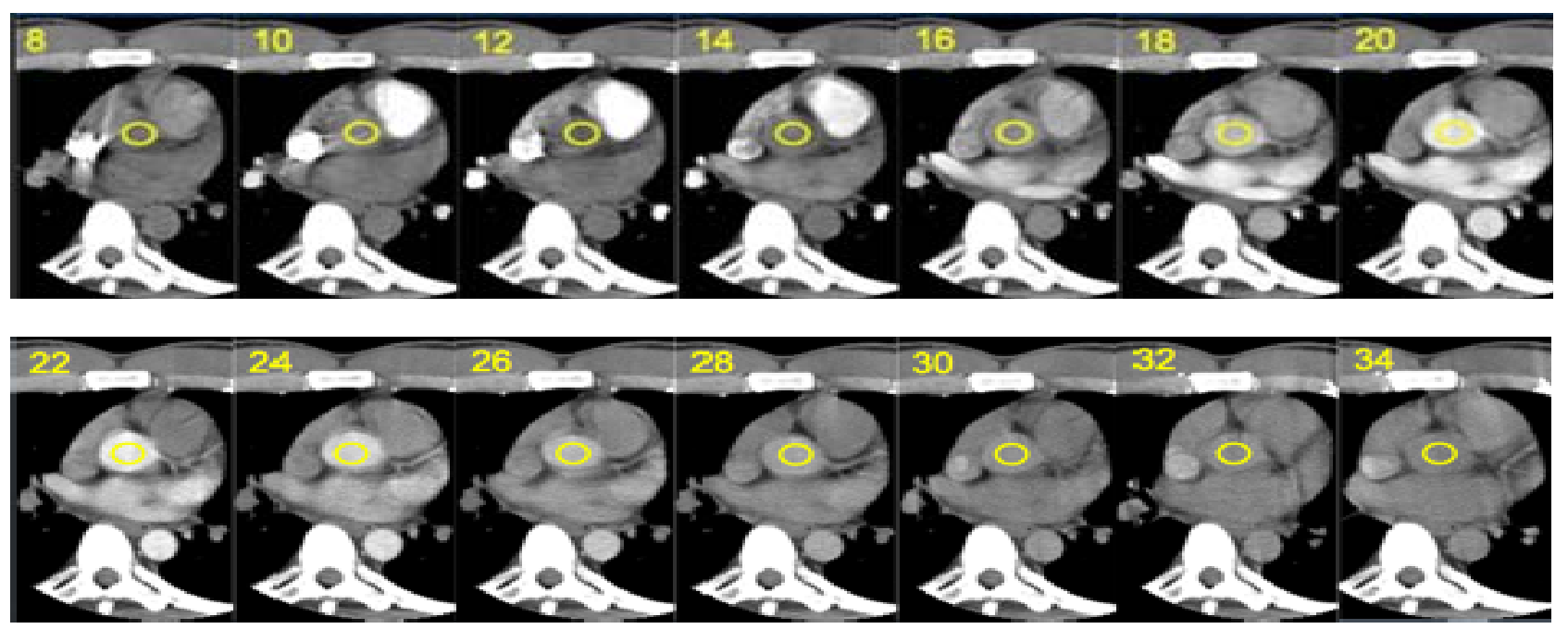

Figure 1. Representative timing test-bolus images with region of interest (yellow circle) beginning at 8 seconds and concluding at 34 seconds with images obtained every 2 seconds at the level of the aortic root. 


\subsection{Transthoracic Echocardiography}

Echocardiographic images (Philips IE-33; Andover, Massachusetts) were analyzed on an Agfa Heartlab (Ridgefield Park, New Jersey) workstation. Echocardiographic data recorded for analysis included left ventricular enddiastolic volume (LVEDV) and left ventricular end-systolic volume (LVESV) by biplane Simpson's method and HR [10]. CO calculated by echo was determined by HR * stroke volume (SV = LVEDV - LVESV).

\subsection{Study Measurement Methodology}

The peak aortic root contrast attenuation (PARCA) was determined by measurement of peak Hounsfield units (HU) in region-of-interest (ROI) analysis within the aortic root for all fourteen images of the transit bolus scan. An area of approximately $60 \%-80 \%$ of the lumen was selected. The ascending aorta cross-sectional area at the time of PARCA was also measured in $\mathrm{cm}^{2}$.

\subsection{Statistical Analysis}

Demographic data are reported as mean \pm standard deviation (SD). Pearson correlation coefficients $(r)$ with twotailed $\mathrm{p}$ values were calculated for the linear relationship between PARCA and CO and also between PARCA and $\mathrm{CI}$ in all patients. After a minimum of four weeks to minimize recall bias, the PARCA measurements were repeated by the original reader for intra-ob server variability assessment and by a second blinded independent reader for inter-observer variability. Correlation was similarly assessed to compare the Stewart-Hamilton-based $\mathrm{CO}$ with the echo-based CO. Based on previous studies by Garrett et al. and Mahnken et al. [6,8], time-enhancement curves were calculated for determination of CO from the test-bolus data via a similar ROI analysis as described above. The time-enhancement curves were transferred to a separate computer equipped with a dedicated software tool for the calculation of CO based on the Stewart Hamilton equation:

$$
\mathrm{CO}=\frac{Q}{\int_{0}^{\infty} c(t) \mathrm{d} t}
$$

where $Q$ is the amount of indicator injected and $c(t)$ is the indicator concentration as a function of time.

Other correlations include the time to PARCA and PARCA upslope: the former was based on the time(s) it took to reach the peak aortic root contrast attenuation, which was calculated depending on which image the maximal HU were obtained with the first image being taken after $8 \mathrm{~s}$ and each following image being taken every $2 \mathrm{~s}$ thereafter; the latter was calculated based on the $\mathrm{HU}$ in the ascending aorta when it peaks divided by the time taken for it to peak. Similar to a previous study [11], PARCA was also correlated to the various LV parameters: LVEF, SV, LVEDV, and LVESV. $p$ values $<0.05$ were considered statistically significant. A linear regression for the relationship between PARCA and CO was also completed. We derived a formula for the estimation of CO based upon these relationships. Pearson correlation coefficients were also performed for analysis of intra-and inter-observer variability. A Bland-Altman analysis was also performed to assess the agreement between the two methods of CO estimation. Statistical calculations were performed using the MedCalc version 11.3 (Mariakerke, Belgium) software package.

\section{Results}

Demographic information for the study cohort is detailed in Table 1.

The mean time between echocardiography and CTCA was $62 \pm 93$ days. As expected due to beta blocker use during CT, HR was lower during CTCA: $59 \pm 11$ compared to $68 \pm 14 \mathrm{bpm}$ with echo $(p<0.001)$.

By echo, mean SV, CO, LVEF and cardiac index (CI) were $67 \pm 19 \mathrm{ml}, 4.5 \pm 1.6 \mathrm{~L} / \mathrm{min}, 61 \pm 6 \%$ and $2.2 \pm 0.7$ $\mathrm{L} / \mathrm{min} / \mathrm{m}^{2}$, respectively. PARCA was $207 \pm 46 \mathrm{HU}$ and correlated to CO and CI with $r=-0.64, p<0.0001$ (Figure 2) and $r=-0.55, p<0.001$ (Figure 3), respectively. Stewart-Hamilton-based CO correlated with $r=0.43, p<$ 0.001 . The linear regression analysis using PARCA estimates $\mathrm{CO}=-0.02 *$ PARCA +9.1 . Results of the Bland Altman analysis are seen in Figure 4.

Table 1. Characteristics of study cohort.

\begin{tabular}{|c|c|}
\hline Features & Value \\
\hline \multicolumn{2}{|l|}{ Sex } \\
\hline Female, n (\%) & $18(52.9 \%)$ \\
\hline Male, n (\%) & $16(47.1 \%)$ \\
\hline Age, $y$, mean $\pm \mathrm{SD}^{*}$ & $58.2 \pm 12.7$ \\
\hline Body Mass Index, $\mathrm{kg} / \mathrm{m}^{2}$, mean $\pm \mathrm{SD}^{*}$ & $29.6 \pm 7.4$ \\
\hline Body Surface Area, $\mathrm{m}^{2}$, mean $\pm \mathrm{SD}^{*}$ & $2.0 \pm 0.3$ \\
\hline \multicolumn{2}{|l|}{ Race } \\
\hline White, n (\%) & $16(47.0 \%)$ \\
\hline Black, n (\%) & $14(41.2 \%)$ \\
\hline Other, n (\%) & $4(11.8 \%)$ \\
\hline \multicolumn{2}{|l|}{ Indications for $\mathrm{CTCA}^{* *}$} \\
\hline Chest Pain, n (\%) & 19 (55.9\%) \\
\hline Other, n (\%) & $4(11.8 \%)$ \\
\hline Dyspnea, n (\%) & $3(8.8 \%)$ \\
\hline Arrythmia, n (\%) & $2(5.9 \%)$ \\
\hline Syncope, n (\%) & $2(5.9 \%)$ \\
\hline Abnormal Stress Test, n (\%) & $2(5.9 \%)$ \\
\hline Aortic Dissection, n (\%) & $2(5.9 \%)$ \\
\hline
\end{tabular}




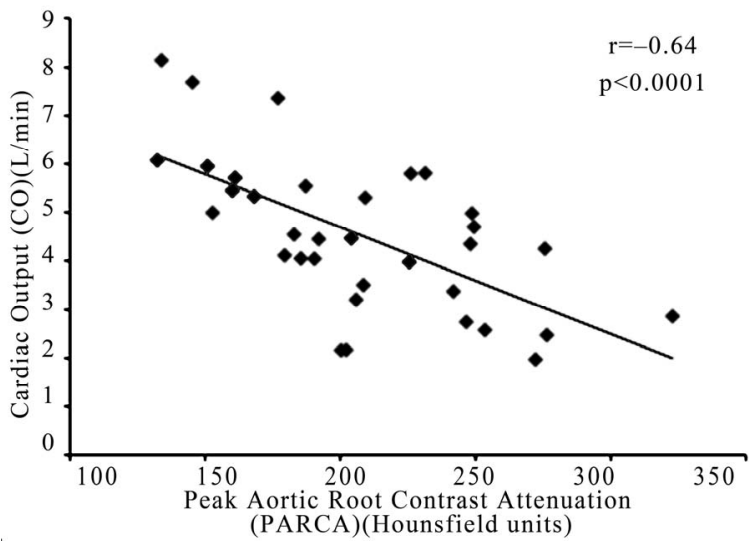

Figure 2. The relationship between peak aortic root contrast attenuation (PARCA) and cardiac output (CO).

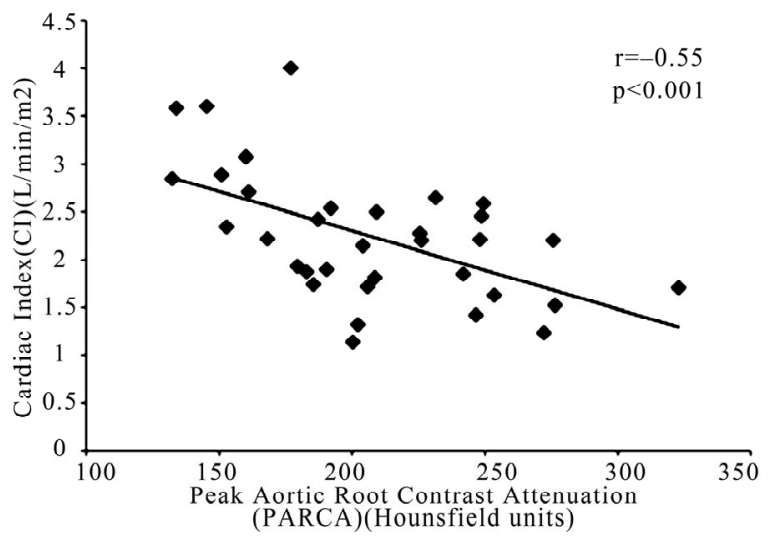

Figure 3. The relationship between peak aortic root contrast attenuation (PARCA) and cardiac index (CI).

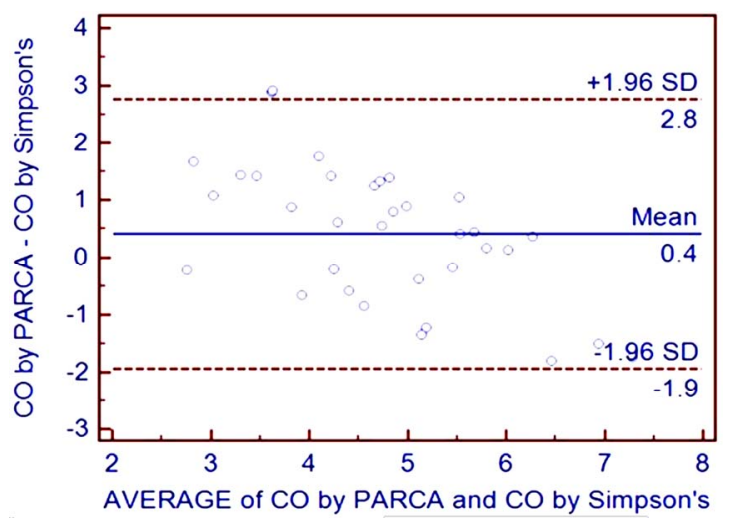

Figure 4. The Bland-Altman relationship between cardiac output (CO) by the PARCA method and cardiac output by the Simpson's biplane method.

Representative timing bolus curves from a high CO and low CO patient are seen in Figures 5(a) and (b), respectively. The intra-observer agreement was determined to be 0.99 by Pearson correlation coefficient for PARCA measurements (Figure 6(a)). Additionally, inter-observer agreement was computed to be 0.95 (Figure 6(b)).

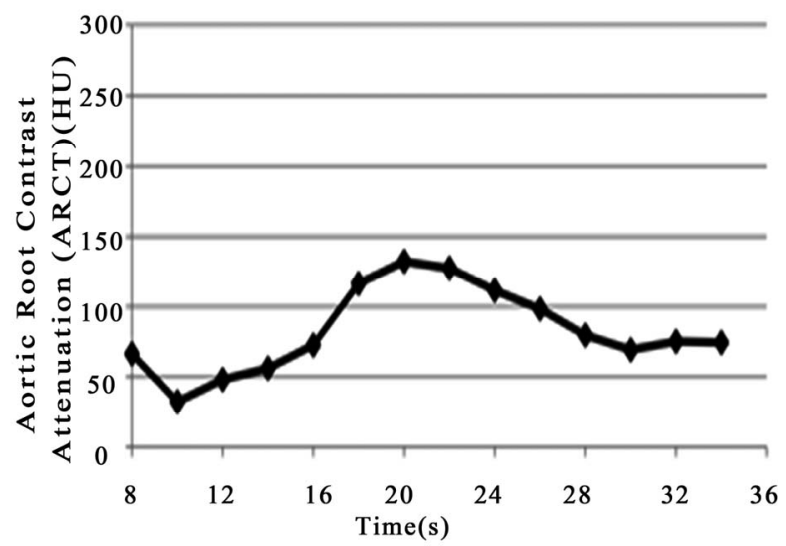

(a)

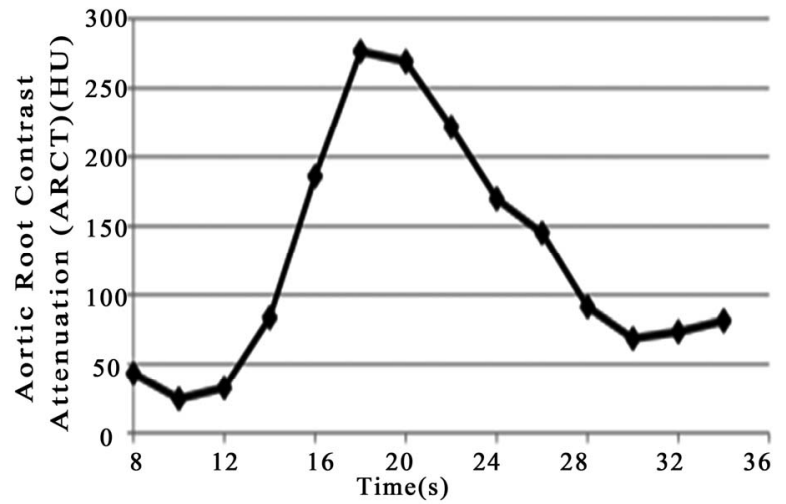

(b)

Figure 5. Representative timing bolus curves for a high cardiac output patient (a) and low cardiac output patient (b).

We tested PARCA against other potential correlates. The time to PARCA was $20.0 \pm 1.8 \mathrm{~s}$ and correlated to CO and CI with $r=-0.13, p=0.46$ and $r=-07, p=0.69$. The PARCA up-slope was $10.3 \pm 2.7 \mathrm{HU} / \mathrm{s}$ and correlated to CO and CI with $r=-0.12, p=0.50$ and $r=-0.15$, $p=0.40$. The PARCA*HR was $12141.4 \pm 3805.8 \mathrm{HU}^{*}$ beats/min and correlated to CO and CI with $r=-0.34$, $p<0.05$ and $r=-0.27, p=0.12$, respectively. The PARCA*Cross Sectional area within the ascending aorta was $1185.8 \pm 407.5 \mathrm{HU}^{*} \mathrm{~cm}^{2}$ and correlated to $\mathrm{CO}$ and CI with $r=-0.24, p=0.17$ and $\mathrm{r}=-0.18, p=0.31$. The PARCA correlated to LVEF with $\mathrm{r}=-0.24, p=0.17$, to SV with $r=-0.61, p<0.001$, to LVEDV with $r=-0.58$, $p<0.001$, and to LVESV with $r=-0.42, p<0.05$.

\section{Discussion}

CTCA has the ability to provide crucial anatomic information about the coronary arteries and myocardium, and physiologic data such as cardiac output. The non-invasive measurement of cardiac output by CT has traditionally required assessment of end-systolic and end-diastolic volumes, acquired during retrospective-gating. In this study, however, we show that prospective CTCA studies 


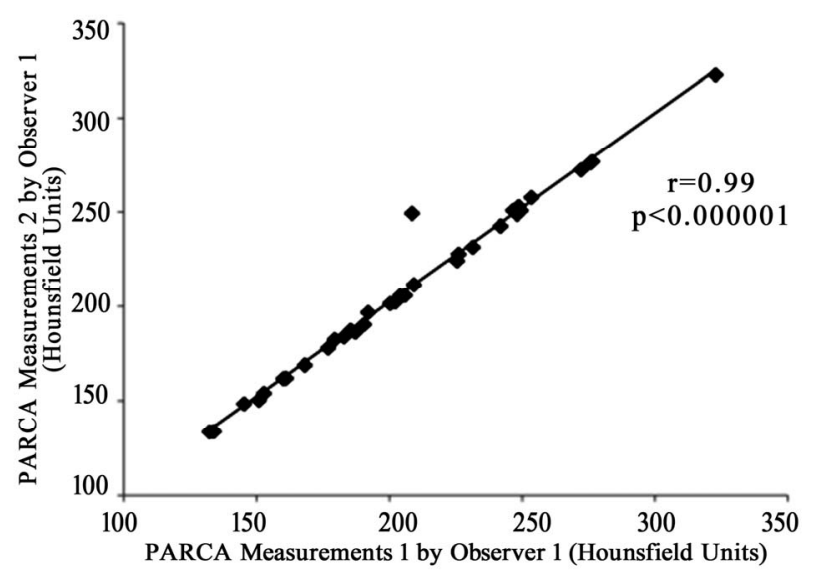

(a)

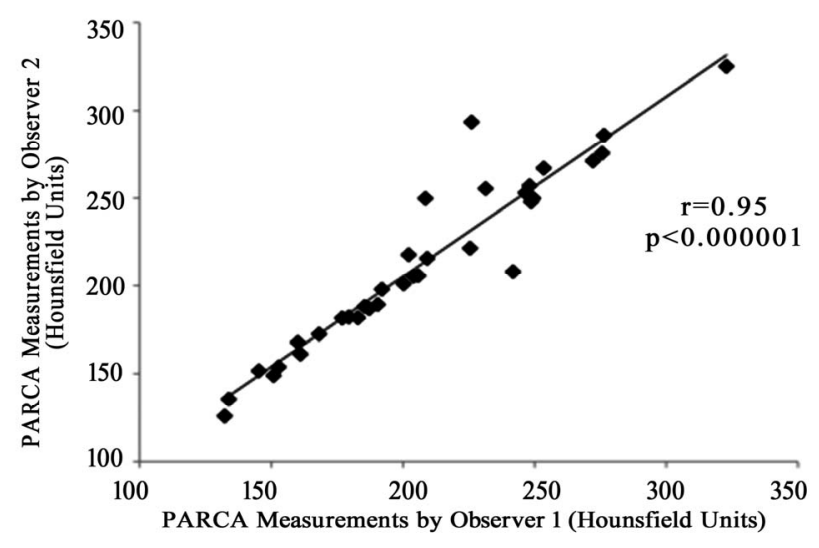

(b)

Figure 6. The intra-observer (a) and inter-observer (b) variability by Pearson correlation coefficient for PARCA measurements.

may retain the ability to predict cardiac output based on contrast dynamics in an easily obtainable and reproducible way. Our study demonstrates that CO is inversely related to PARCA (Figure 2). Similar results were observed after accounting for body size (Figure 3). As preclinical models suggest $[9,12]$ the most important patient-related factor affecting the timing of contrast enhancement is cardiac output. As seen in Figures 5(a) and (b), the circulation of contrast medium slows and the magnitude of peak aortic enhancement increases with a reduction in $\mathrm{CO}[9,12]$.

Coupled with other techniques [13], CT may provide more comprehensive non-invasive hemodynamic assessment, which could broaden the applications of this technology. Furthermore, PARCA is easily reproducible and does not require complex software algorithms. The value of cardiac CT may increase as it replaces more of the indications for invasive angiography.

\section{Limitations}

Our study has several limitations. As there was no docu- mented change in clinical status, we assumed that the hemodynamic status was comparable for both studies. Being a retrospective analysis, the CTCA and echocardiography studies were not performed concomitantly and the temporal proximity between the two studies was not ideal.

We compared two different noninvasive methods in this study; however, invasive measurement by a cardiac catheterization would provide the most accepted gold standard assessment. As mentioned above, the HR was lower during the CTCA vs. echo, which may affect the results - cardiac output may have decreased as a result of beta blocker administration for the CTCA, but as beta blockade was consistently applied for the patients in the study, it may not change the effect on correlation to echo-determined cardiac output. Also, our findings are limited to patients without significant cardiac output abnormalities, and the number of patients with significantly decreased ejection fraction was limited. In a prior study evaluating cardiopulmonary transit time with MR angiography [14], the transit time was increased with decreased LVEF; however, poor temporal resolution with timing bolus by CT may prevent the verification of such a finding.

\section{Conclusion}

This novel method for CO estimation by CTCA appears feasible. The CT physiologic parameters using the timing test-bolus data moderately correlated with echocardiographic assessment of cardiac output. The clinical usefulness of this parameter is uncertain in the absence of further clinical validation. Nevertheless, the calculation of $\mathrm{CO}$ adds important hemodynamic data to anatomic information provided by CTCA, and further development of this method may preserve assessment of left ventricular performance in prospective triggering.

\section{Acknowledgements}

Study supported by W.T. Gill, Jr. Research Fellowship, George Washington University and The Richard B. and Lynne V. Cheney Cardiovascular Institute, George Washington University.

\section{REFERENCES}

[1] G. M. Pohost, L. Hung and M. Doyle, "Clinical Use of Cardiovascular Magnetic Resonance,” Circulation, Vol. 108, No. 6, 2003, pp. 647-653. doi:10.1161/01.CIR.0000083233.86078.3E

[2] J. D. Schuijf, J. J. Bax, L. P. Salm, J. W. Jukema, H. J. Lamb, E. E. van der Wall and A. de Roos, "Noninvasive Coronary Imaging and Assessment of Left Ventricular Function Using 16-Slice Computed Tomography,” American Journal of Cardiology, Vol. 95, No. 5, 2005, pp. 571-574. 
doi:10.1016/j.amjcard.2004.11.002

[3] S. M. Chang, S. Bhatti and F. Nabi, "Coronary Computed Tomography Angiography,” Current Opinion in Cardiology, Vol. 26, No. 5, 2011, pp. 392-402.

doi:10.1097/HCO.0b013e32834938c6

[4] G. L. Raff, K. M. Chinnaiyan, D. A. Share, T. Y. Goraya, E. A. Kazerooni, M. Moscucci, R. E. Gentry and A. Abidov, "Radiation Dose from Cardiac Computed Tomography Before and after Implementation of Radiation Dose-Reduction Techniques,” Journal of American Medical Association, Vol. 301, No. 22, 2009, pp. 2340-2348. doi:10.1001/jama.2009.814

[5] J. Conway and P. Lund-Johansen, "Thermodilution Method for Measuring Cardiac Output," European Heart Journal, Vol. 11, No. 1, 1990, pp. 17-20.

[6] A. H. Mahnken, E. Klotz, A. Hennemuth, B. Jung, R. Koos, J. E. Wildberger and R. W. Gunther, "Measurement of Cardiac Output from a Test-Bolus Injection in Multislice Computed Tomography," European Radiology, Vol. 13, No. 11, 2003, pp. 2498-2504. doi:10.1007/s00330-003-2054-X

[7] R. J .Herfkens, L. Axel, M. J. Lipton, S. Napel, W. Berninger and R. Redington, "Measurement of Cardiac Output by Computed Transmission Tomography," Investigative Radiology, Vol. 17, No. 6, 1982, pp. 550-553. doi:10.1097/00004424-198211000-00005

[8] J. S. Garrett, P. Lanzer, W. Jaschke, E. Botvinick, R. Sievers, C. B. Higgins and M. J. Lipton, "Measurement of Cardiac Output by Cine Computed Tomography,” American Journal of Cardiology, Vol. 56, No. 10, 1985, pp. 657-661. doi:10.1016/0002-9149(85)91030-6
[9] K. T. Bae, J. P. Heiken and J. A. Brink, “Aortic and Hepatic Contrast Medium Enhancement at CT. II. Effect of Reduced Cardiac Output in a Porcine Model,” Radiology, Vol. 207, No. 3, 1998, pp. 657-662.

[10] J. H. McGowan and J. G. F. Cleland, "Reliability of Reporting Left Ventricular Systolic Function by Echocardiography: A Systematic Review of 3 Methods,” American Heart Journal, Vol. 146, No. 3, 2003, pp. 388-397. doi:10.1016/S0002-8703(03)00248-5

[11] N. E. Manghat, G. J. Morgan-Hughes, S. R. Shaw, A. J. Marshall and C. A. Roobottom, "Impaired Left Ventricular Function Has a Detrimental Effect on Image Quality in Multi-Detector Row CT Coronary Angiography," Clinical Radiology, Vol. 63, No. 4, 2008, pp. 415-423. doi:10.1016/j.crad.2007.08.019

[12] K. T. Bae, "Intravenous Contrast Medium Administraction and Scan Timing at CT: Considerations and Approaches," Radiology, Vol. 256, No. 1, 2010, pp. 32-61. doi:10.1148/radiol.10090908

[13] R. S. Dusaj, K. C. Michelis, M. Terek, R. Sanai, R. Mittal, J. F. Lewis, R. K. Zeman and B. G. Choi, "Estimation of Right Atrial and ventricular Hemodynamics by CT Coronary Angiography,” Journal of Cardiovascular Computed Tomography, Vol. 5, No. 1, 2011, pp. 44-49. doi:10.1016/j.jcct.2010.10.005

[14] S. M. Shors, W. G. Cotts, B. Pavlovic-Surjancev, C. J. Francois and J. P. Finn, "Heart Failure: Evaluation of Cardiopulmonary Transit Times with Time-Resolved MR Angiography,” Radiology, Vol. 229, No. 3, 2003, pp. 743-748. doi:10.1148/radiol.2293021363 